\title{
Pais e/ou Professores? Acordo entre Informadores na Avaliação Socioemocional de Pré-Escolares ${ }^{1}$
}

\author{
Sofia Major ${ }^{2}$ \\ Maria João Seabra-Santos \\ Faculdade de Psicologia e de Ciências da Educação Universidade de Coimbra
}

\begin{abstract}
RESUMO - Pais e professores são os informadores mais requisitados na avaliação socioemocional em idade pré-escolar. Todavia, o facto de informadores que interagem com a criança em diversos contextos poderem ter perspetivas distintas coloca, frequentemente, em causa a fiabilidade e validade desses relatos. O presente artigo tem por objetivo rever 22 estudos de 57 identificados, publicados desde 1987 até 2011, acerca do acordo entre informadores dos contextos familiar e escolar, no preenchimento de inventários comportamentais. Os vários estudos sugerem um grau de acordo reduzido a moderado e a inexistência de um informador-chave na avaliação socioemocional, alertando para a necessidade de recolha paralela da perspetiva de pais e professores, para obter um retrato mais completo dos comportamentos. São discutidas as implicações dos resultados para estudos futuros.
\end{abstract}

Palavras-chave: pais, professores, acordo entre informadores, avaliação socioemocional, pré-escolar

\section{Parents and/or Teachers? Inter-Ratter Agreement on Preschoolers Social- Emotional Assessment}

\begin{abstract}
Parents and teachers are the most requested informants for preschool social-emotional assessment. However, the fact that informants who interact with the child in different settings may have different perspectives frequently calls into question the reliability and validity of these reports. The present paper intends to review 22 studies of 57 identified, published between 1987 and 2011, about the agreement between informants from home and school settings while completing rating scales. The studies suggest a low to moderate informant agreement and the inexistence of a key-informant in social-emotional assessment, therefore drawing attention to the need to collect the perspectives of both parents and teachers, as a means of achieving a more complete picture of behaviors. Results are discussed in their implications for future research.
\end{abstract}

Keywords: parents, teachers, inter-ratter agreement, social-emotional assessment, preschool

$\mathrm{Na}$ tentativa de ultrapassar as capacidades ainda limitadas ao nível cognitivo e linguístico das crianças em idade pré-escolar, o recurso a diferentes informadores na avaliação psicológica destas crianças reveste-se de uma importância particular (Crane, Mincic, \& Winsler, 2011; Major, 2011). Assim, a inviabilidade de utilizar medidas de auto-relato chama a atenção para a necessidade de incorporar a informação facultada por pais e educadores de infância (designação utilizada para os professores do ensino préescolar) no processo de avaliação psicológica no período pré-escolar. Por outro lado, contrariamente à avaliação do funcionamento cognitivo, levada a cabo através de uma abordagem direta à criança, os problemas e as competências comportamentais daquela são, tendencialmente, identificados e descritos através dos relatos de outros significativos (Crane et al., 2011; Keogh \& Bernheimer, 1998). Acresce

1 Apoio: Artigo elaborado a partir da tese de doutorado da primeira autora, Avaliação de aptidões sociais e problemas de comportamento em idade pré-escolar: Retrato das crianças portuguesas, apresentada à Faculdade de Psicologia e de Ciências da Educação da Universidade de Coimbra (Portugal), com financiamento de uma bolsa de doutorado da FCT (SFRH/BD/29141/2006).

2 Endereço para correspondência: Faculdade de Psicologia e de Ciências da Educação, Rua do Colégio Novo, Apartado 6153, 3001-802 Coimbra, Portugal. E-mail: smajor@fpce.uc.pt que, enquanto os adultos procuram apoio/auxílio por si próprios, no caso das crianças a decisão da necessidade de uma avaliação/acompanhamento psicológico fica, na maioria dos casos, a cargo dos pais ou dos professores (Gimpel \& Holland, 2003; Stanger \& Lewis, 1993). Em conformidade com estas ordens de razões, diversos autores (Klyce, Conger, Conger, \& Dumas, 2011; Kroes, 2006; Renk, 2005) reforçam a importância de recorrer a múltiplos informadores e métodos na avaliação destas crianças, dada a instabilidade/especificidade do comportamento da criança nos diversos contextos de interação, diferentes expetativas dos informadores e diferenças no tipo de comportamento observado.

É, pois, relativamente consensual a necessidade e importância de obter as perspetivas de várias fontes (e.g., pais, educadores/professores, psicólogos ou outros técnicos) (Achenbach, McConaughy, \& Howell, 1987; Grietens et al., 2004; Kamphaus \& Frick, 1996; Klyce et al., 2011; Kroes, 2006; Renk, 2005; Winsler \& Wallace, 2002) no processo de avaliação socioemocional de crianças pré-escolares. Esta ideia da recolha de informação junto de múltiplos informadores tem subjacente a importância das diferentes perspetivas de cada um deles, na tentativa de integrar essa informação para obter um retrato mais coerente/fidedigno do funcionamento da criança, ao interagir com vários adultos 
com um papel significativo na sua vida e nos diversos contextos em que passa a maior parte do seu tempo (casa e jardim-de-infância) (Achenbach \& McConaughy, 1987; Gagnon, Nagle, \& Nickerson, 2007; Murray et al., 2007; Offord et al., 1996; Verhulst \& Akkerhuis, 1989).

A importância da cooperação e participação dos pais e de professores no fornecimento de informações no decorrer do processo avaliativo de uma criança em idade pré-escolar é ainda sublinhada dado ter um impacto significativo na procura de auxílio (Herrera \& Little, 2005) na medida em que, quando os pais veem as suas queixas acerca dos comportamentos de agressividade, atividade excessiva e desobediência corroboradas por professores ou auxiliares, mais facilmente procurarão apoio psicológico.

Neste contexto, por volta dos anos 70 do século XX, as escalas de avaliação obtiveram uma grande aceitação por parte da comunidade científica e, hoje em dia, representam um dos instrumentos mais utilizados na avaliação do comportamento das crianças (e.g., dada a sua rapidez de aplicação e reduzido custo) (Liu, Cheng, \& Leung, 2011). Todavia, rapidamente lhes ficou associada a limitação de baixas a modestas correlações obtidas para o acordo entre informadores, gerando uma certa apreensão acerca da fiabilidade e validade dos resultados alcançados com estes instrumentos (Renk, 2005).

Por conseguinte, quer os benefícios, quer as contrariedades em trabalhar com informação proveniente de diversas fontes têm despertado o interesse tanto ao nível da investigação como no trabalho clínico com crianças e respetivas famílias (Grietens et al., 2004; Renk, 2005). Ou seja, se por um lado o recurso a vários informadores permite recolher informação única acerca do funcionamento socioemocional das crianças, por outro lado, estas diferentes perceções poderão tornar-se problemáticas para profissionais de saúde mental interessados no padrão habitual de funcionamento da criança (Klyce et al., 2011; Renk, 2005; Verhulst \& Akkerhuis, 1989), e até colocar dúvidas acerca da fidedignidade das classificações atribuídas pelos informadores (Gagnon et al., 2007; Renk, 2005; Seabra-Santos \& Gaspar, 2012). Consequentemente, várias questões podem ser equacionadas: o que fazer quando dois informadores não concordam, ou ainda, quem está numa melhor posição para determinar se o problema é relevante ou não? Finalmente, quem faculta informação mais precisa e válida acerca dos comportamentos das crianças? (Kroes, 2006).

A revisão da literatura especializada permite-nos constatar que a existência de discrepâncias entre informadores representa mais a regra do que a exceção. Neste sentido, encontra-se repetidamente referência ao reduzido a moderado acordo entre informadores nos relatos de problemas de comportamento (e.g., Achenbach et al., 1987; Gagnon, Vitaro, \& Tremblay, 1992; Gross, Fogg, Garvey, \& Julion, 2004; Offord et al., 1996). Convém, no entanto, salientar que a vasta maioria dos estudos encontrados acerca do acordo entre pais e professores se reporta a crianças em idade escolar, o que deixa a compreensão deste fenómeno menos esclarecida para a população pré-escolar (Crane et al., 2011; Grietens et al., 2004; Gross et al., 2004; Herrera \& Little, 2005; Phillips \& Lonigan, 2010; Winsler \& Wallace, 2002). Por outro lado, é importante sublinhar que, enquanto o acordo entre informadores no que diz respeito à avaliação de problemas de comportamento tem sido amplamente estudado (e.g., Achenbach et al., 1987), já a competência social não tem sido alvo da mesma atenção (Crane et al., 2011; Renk, 2005; Renk \& Phares, 2004).

\section{Pais ou professores?}

Num primeiro momento, a maioria dos estudos acerca do funcionamento socioemocional das crianças focava-se apenas num informador, apontando alguns deles os pais como a fonte de informação prevalecente, enquanto outros atribuíam esse papel aos professores (Treutler \& Epkins, 2003). Porém, hoje em dia, pais e professores são tradicionalmente considerados como os informadores que, em conjunto, poderão desempenhar um papel mais importante na avaliação comportamental e/ou emocional das crianças. Esta evidência é justificada pelo facto de serem os adultos com quem as crianças passam mais tempo, com mais oportunidades para as observar e num leque de situações mais diversificado (BergNielsen, Solheim, Belsky, \& Wichstrom, 2012; Grietens et al., 2004; Keogh \& Bernheimer, 1998; Vitaro, Gagnon, \& Tremblay, 1991). Deste modo, dado que o comportamento da criança numa situação de avaliação em consultório poderá não ser representativo dos problemas que motivaram o seu encaminhamento para a consulta, tem sido enfatizada a relevância de uma avaliação de problemas de comportamento sustentada numa perspetiva conjunta de pais e professores, por forma a obter descrições do comportamento em diferentes contextos e a aumentar a validade dos dados recolhidos (Achenbach et al., 1987; Gagnon et al., 1992; Gagnon et al., 2007; Herrera \& Little, 2005).

\section{Os pais enquanto informadores}

Os pais tendem a ser considerados bons informadores acerca do funcionamento socioemocional dos seus filhos e, geralmente, são capazes de fornecer informações detalhadas acerca dos comportamentos (competências e problemas), uma vez que passam muito tempo com estes, sendo quem está mais a par do comportamento da criança ao longo do tempo e situações (Culp, Howell, Culp, \& Blankemeyer, 2001; Klyce et al., 2011). Neste sentido, as figuras parentais estão, em geral, capacitadas para oferecer informação única acerca dos seus filhos, constituindo a melhor fonte de informação no que diz respeito à história de vida da criança, às dificuldades atuais e aos fatores externos com influência no comportamento desta (e.g., falecimento de um familiar, mudança recente de residência) (Gimpel \& Holland, 2003). Por outro lado, a inclusão dos pais no processo avaliativo enfatiza a importância de envolver aqueles que são os mais afetados pela situação da criança num processo colaborativo de desenvolvimento de um programa interventivo (Hundert, Morrison, Mahoney, Mundy, \& Vernon, 1997).

Neste contexto, a mãe é frequentemente o informador mais disponível para fornecer dados acerca dos comportamentos da criança (Treutler \& Epkins, 2003), pelo que a coleta da sua perspetiva é fulcral, tanto em termos de prática clínica, 
como ao nível da investigação. Com efeito, se o contacto mais intenso e a longo prazo que a mãe partilha com a criança lhe permite ter acesso a um reportório de informação acerca do comportamento daquela que é inacessível à maioria dos demais informadores, acresce, ainda, que o preenchimento de um (ou vários) questionário(s) se revela, na maioria dos casos, uma estratégia de custo nulo na coleta de informação para estudos epidemiológicos (Major, 2011).

Ao analisar a literatura disponível acerca do acordo entre informadores, um dado que se impõe consiste na sub-representação de participação da figura paterna, comparativamente à materna. Assim, nas revisões da literatura sobre psicopatologia do desenvolvimento denotase que prevalecem taxas de participação da figura paterna muito reduzidas. A título de exemplo, Phares e Compas (1992) analisaram a inclusão do pai nas investigações clínicas acerca de crianças e adolescentes realizadas entre 1984-1991 e constataram que, dos 577 artigos estudados, 277 (48.0\%) incluíam apenas a mãe contra $8(1.4 \%)$ que envolviam somente a figura paterna. Apesar de alguns ganhos neste domínio em estudos sobre a criança, esta realidade mantinhase, ainda, 13 anos mais tarde, como comprova a revisão da literatura levada a cabo por Phares, Fields, Kamboukos e Lopez (2005) segundo a qual, dos 514 estudos revistos, 231 $(45.0 \%)$ envolviam apenas a mãe como informador acerca do comportamento da criança, contra 11 (2.1\%) com foco exclusivo na figura paterna.

Neste contexto, e apesar da importância de recolher informação junto de ambas as figuras parentais, perante a dificuldade de envolver ambos os pais e o professor no processo avaliativo, Grietens et al. (2004) recomendam a coleta de informação junto de pelo menos um dos progenitores e do professor.

\section{Os professores enquanto informadores}

No que diz respeito às competências académicas das crianças, os professores são reconhecidos como fontes importantes na avaliação, na medida em que os dados por eles facultados são, frequentemente, relevantes para encaminhar a criança para serviços de educação especial, inclusão num grupo de intervenção ou para informar os pais e outros profissionais acerca dos desempenhos daquela na escola (Mashburn, Hamre, Downer, \& Pianta, 2006). Porém, a perspetiva dos professores acerca do funcionamento socioemocional dos seus alunos nem sempre foi tida em consideração no passado (Kamphaus \& Frick, 1996), focando-se as investigações sobre a avaliação socioemocional em idade pré-escolar em entrevistas e escalas de avaliação preenchidas pelos pais.

No entanto, dado o crescente número de crianças a frequentar instituições pré-escolares, a perspetiva de professores e auxiliares tem vindo a ganhar uma importância acrescida (Hinshaw, Han, Erhardt, \& Huber, 1992). Vários fatores se encontram associados ao aumento do interesse pela avaliação do funcionamento socioemocional das crianças em contexto escolar (Kamphaus \& Frick, 1996). Em primeiro lugar destaca-se o facto de ser o contexto onde a criança passa muito do seu tempo e onde é confrontada com múltiplas exigências/requisitos (e.g., ficar sentada, interagir com os pares) que poderão constituir desafios inexistentes noutros contextos e em relação aos quais ela poderá apresentar dificuldades. Achenbach (1991) acrescenta, ainda, outros argumentos favoráveis à coleta de informação em contexto escolar, classificado por este autor como uma autêntica "arena desenvolvimental". Assim, é referido, por um lado, o facto de os professores serem geralmente os segundos adultos com um papel mais influente na vida das crianças (logo a seguir aos pais), cuja experiência, treino e oportunidades de observação lhes permitem apreender características do funcionamento que não são observáveis pelos pais. Por outro lado, salienta-se a circunstância de os seus relatos serem isentos da influência das dinâmicas familiares (apesar de sujeitos às dinâmicas interpessoais da escola).

Por conseguinte, os professores assumem um papel de destaque enquanto observadores ditos "naturais" em contexto escolar, uma vez que observam muitas crianças ao longo do tempo e em situações sociais diversas (e.g., escola, visitas de estudo). Estes informadores têm, ainda, a clara vantagem de observar a criança num contexto estruturado e de terem um grupo de comparação (turma) com o qual podem confrontar o comportamento da criança referenciada (Grietens et al., 2004; Kamphaus \& Frick, 1996; Mashburn et al., 2006; Milfort \& Greenfield, 2002; Verhulst \& Akkerhuis, 1989). Por outro lado, podem, igualmente, observar a criança por períodos extensos de tempo em interação com os pares (cerca de 6 horas por dia, durante 5 dias da semana), o que lhes permite aceder a uma perspetiva única sobre comportamentos sociais (Kroes, 2006; Mashburn et al., 2006; Milfort \& Greenfield, 2002) e podendo detetar eventuais problemas aos níveis académico ou social, mais difíceis de percecionar por parte dos pais (Verhulst \& Akkerhuis, 1989).

Relativamente à idade pré-escolar, os relatos dos professores revestem-se de importância acrescida na validação dos problemas de comportamento, uma vez que nesta faixa etária a amplitude dos comportamentos de desobediência e agressão é relativamente vasta (Keenan \& Wakschlag, 2000). Por conseguinte, todas estas vantagens, somadas aos reduzidos custos e pouco tempo necessário para a recolha de informação junto de professores, tornam a possibilidade de obter dados a partir destes informadores como uma das opções de primeira linha na avaliação socioemocional de crianças (Mashburn et al., 2006).

Neste sentido, e dada a relevância da reflexão acerca da questão do acordo entre informadores em contexto familiar e escolar, o presente artigo visa apresentar uma revisão dos estudos empíricos publicados entre 1987 e 2011, com referência não só a problemas de comportamento, mas também às competências das crianças em idade pré-escolar, tendo dois objetivos principais: (a) sintetizar os resultados dos estudos realizados em torno desta temática, desde a publicação da meta-análise de Achenbach et al. (1987), tomada como limite temporal inferior até ao presente; e (b) esclarecer e identificar a existência de padrões comuns de resultados nos vários estudos considerados, assim como contradições, lacunas e inconsistências, com vista a apresentar sugestões para estudos futuros. 


\section{Método}

\section{Localização e seleção dos estudos}

Foram efetuadas diversas pesquisas em bases de dados representativas ao nível das Ciências Sociais e Humanas: ProQuest, OvidSP e B-On: Online Knowledge Library-Search. A busca foi realizada através de combinações diferentes das seguintes palavras-chave: preschool, agreement, inter-rater, informants, parent-teacher, social-emotional, behavior. A meta-análise de Achenbach et al. (1987), que constitui um estudo clássico nesta área, foi tomada como limite inferior do período de estudos a considerar na revisão. Os estudos foram integrados na revisão caso obedecessem aos seguintes critérios de inclusão: (a) artigos com foco na questão do acordo entre informadores na avaliação socioemocional (aptidões sociais e/ou problemas de comportamento); (b) que abrangessem crianças sem atraso de desenvolvimento, com idades dos 3 aos 6 anos (estudos longitudinais com pelo menos uma avaliação no período pré-escolar ou com faixas etárias mais alargadas foram, também, incluídos); (c) sendo as crianças avaliadas por pais e respetivos professores; (d) com recurso a escalas de avaliação; e (e) estando os estudos publicados em revistas com revisão por pares (de forma a garantir a qualidade dos estudos revistos).

Uma vez efetuada a pesquisa bibliográfica foi identificado um total de 57 estudos, dos quais 35 foram excluídos da análise. Destes, seis foram descartados por serem de natureza teórica, três por se referirem a crianças com perturbações do desenvolvimento, três por não abrangerem o período préescolar, cinco por analisarem outras questões relacionadas com a utilidade dos informadores e não a questão do acordo entre eles, dez por se referirem a outros informadores (e.g., acordo interparental), e oito por abordarem outros tópicos da avaliação socioemocional (e.g., estudos de adaptação de instrumentos). Cada um dos restantes 22 estudos selecionados foi analisado e resumido atendendo à seguinte informação: autores e ano de publicação, objetivos, dimensão da amostra, idade/ano de escolaridade das crianças, principais resultados e conclusões (cf. Tabela 1).

\section{Caracterização dos estudos}

Dos 22 estudos considerados, 20 apresentam dados relativos à dimensão das amostras, que oscilam entre 33 e 14.811 crianças, sendo que 16 estudos $(72.7 \%)$ incluem mais de 100 participantes. Atendendo ao critério de incluir estudos longitudinais com pelo menos uma avaliação no préescolar ou com faixas etárias mais alargadas (com inclusão do período pré-escolar), as idades dos participantes situam-se entre o ano e meio e os 19 anos, com 54.5\% dos estudos a focarem-se exclusivamente na idade pré-escolar (dos 3 aos 6 anos de idade). Todas as crianças foram avaliadas por pais e respetivos professores, sendo que 13 estudos não apresentam informação acerca de qual o informador em contexto familiar que procedeu à avaliação. Dos nove estudos que apresentam esses dados, as mães são os informadores mais disponíveis (com 65.0 a $92.8 \%$ de participação) comparativamente aos pais (que apenas preencheram 6.0 a 18.5\% dos questionários). Apenas quatro estudos apresentam alguma informação acerca do informador em contexto escolar (e.g., grau académico). A maioria dos estudos envolve amostras da comunidade $(81.8 \%)$.

Todos os estudos utilizaram escalas de avaliação devidamente estandardizadas e validadas, tais como o Teacher Report Form (TRF; Achenbach, 1991) ou as Preschool and Kindergarten Behavior Scales - Second Edition (PKBS-2; Merrell, 2002). Em quatro estudos houve ainda lugar à utilização de outros instrumentos de avaliação, nomeadamente: medidas de observação direta (dois estudos), medidas sociométricas (um estudo) e entrevista (um estudo). Relativamente à problemática avaliada, 11 estudos referem-se à avaliação de problemas de comportamento, três apresentam um foco em aptidões sociais e oito estudos abordam ambos (aptidões sociais e problemas de comportamento). A totalidade dos estudos encontra-se publicada em revistas com revisão por pares, nomeadamente, Journal of Abnormal Child Psychology, Journal of Child and Family Studies, Journal of Psychopathology and Behavioral Assessment e Psychological Bulletin.

\section{Resultados}

De forma a sintetizar a informação disponível relativa aos estudos acerca do acordo entre pais e professores, a Tabela 1 apresenta por ordem cronológica as 22 investigações consideradas para a presente revisão. É importante destacar o crescente número de estudos em redor desta temática que tem vindo a ser publicado, com um estudo na década de 80 (após a publicação da meta-análise de Achenbach et al., 1987) e 14 entre 2000 e 2010.

A análise das pesquisas sintetizadas na Tabela 1 permite salientar vários aspetos. Em primeiro lugar, as correlações reduzidas a modestas encontradas nos vários estudos apontam para um grau de acordo relativamente baixo entre informadores com papéis diferentes na vida da criança (pais e professores) (Achenbach et al., 1987; Cranes et al., 2011; Culp et al., 2001; Gagnon et al., 1992; Gagnon et al., 2007; Goodman, 2001; Gross et al., 2004; Hinshaw et al., 1992; Klyce et al., 2011; Liu et al., 2011; Miner \& Clarke-Stewart, 2008; Murray et al., 2007; Phillips \& Lonigan, 2010; Renk \& Phares, 2004; Stefan, Balaj, Porumb, Albu, \& Miclea, 2009; Stone, Otten, Engels, Vermulst, \& Janssens, 2010; Verhulst \& Akkerhuis, 1989; Vitaro et al., 1991; Winsler \& Wallace, 2002). Em segundo lugar, os pais (em especial a mãe) tendem a cotar os seus filhos com mais problemas de comportamento do que os professores (Cranes et al., 2011; Culp et al., 2001; Gagnon et al., 1992; Gross et al., 2004; Herrera \& Little, 2005; Miner \& Clarke-Stewart, 2008; Verhulst \& Akkerhuis, 1989; Winsler \& Wallace, 2002). Por outro lado, existe alguma evidência segundo a qual pais e professores tendem a concordar mais nas cotações de problemas de comportamento exteriorizados (mais visíveis) do que interiorizados (menos percetíveis) (Achenbach et al., 1987; Gagnon et al., 1992; Gagnon et al., 2007; Goodman, 2001; Hinshaw et al., 1992; Stone et al., 2010; TarrenSweeney, Hazell, \& Carr, 2004; Verhulst \& Akkerhuis, 1989; 
Tabela 1. Sumários dos Estudos Revistos: Acordo entre Informadores dos Contextos Familiar e Escolar

\begin{tabular}{|c|c|c|c|c|c|}
\hline $\begin{array}{c}\text { Autores } \\
\text { Ano }\end{array}$ & Objetivos & $\begin{array}{c}N \\
\text { Amostra }\end{array}$ & $\begin{array}{c}\text { Idade ou } \\
\text { Escolaridade }\end{array}$ & Principais Resultados & Conclusões \\
\hline $\begin{array}{l}\text { Achenbach, } \\
\text { McConaughy, \& } \\
\text { Howell (1987) }\end{array}$ & $\begin{array}{l}\text { Estudo de meta-análise } \\
\text { Analisar o acordo entre } \\
\text { informadores nas cotações de } \\
\text { problemas de comportamento } \\
\text { e emocionais }\end{array}$ & $\begin{array}{c}269 \\
\text { amostras }\end{array}$ & $1^{1 / 2}$ aos 19 anos & $\begin{array}{l}\text { Correlação para o acordo entre pais-professores }=.27 \\
\text { Correlação média para informadores entre contextos }=.28\end{array}$ & $\begin{array}{l}\text { 1. O grau de acordo entre informadores que } \\
\text { desempenham papéis distintos e observam a } \\
\text { criança em diferentes situações é reduzido }\end{array}$ \\
\hline $\begin{array}{l}\text { Verhulst \& } \\
\text { Akkerhuis (1989) }\end{array}$ & $\begin{array}{l}\text { Analisar o grau de acordo } \\
\text { pais-professores quanto a } \\
\text { problemas comportamentais } \\
\text { ou emocionais }\end{array}$ & 1.161 & 4 aos 12 anos & $\begin{array}{l}\text { Maior grau de acordo para problemas exteriorizados face } \\
\text { aos interiorizados } \\
\text { Maior grau de acordo para crianças a receber apoio/ } \\
\text { educação especial }(r=.57) \text { do que para crianças a } \\
\text { frequentar a educação regular }(\mathrm{r}=.30) \\
\text { Para os 4-5 anos: } r=.27 \\
\text { Para os 6-12 anos: } r=.35\end{array}$ & $\begin{array}{l}\text { 1. Grau de acordo reduzido a moderado } \\
\text { 2. Pais apontam mais problemas de } \\
\text { comportamento em comparação com os } \\
\text { professores } \\
\text { 3. Maior grau de acordo para crianças mais } \\
\text { velhas } \\
\text { 4. Da análise dos itens, os professores } \\
\text { cotam as crianças com mais problemas de } \\
\text { relacionamento com os pares ou que interfiram } \\
\text { no funcionamento académico } \\
\text { 5. Da análise dos itens, os pais cotam os filhos } \\
\text { com mais problemas de tipo exteriorizado }\end{array}$ \\
\hline $\begin{array}{l}\text { Vitaro, Gagnon, } \\
\text { \& Tremblay } \\
(1991)\end{array}$ & $\begin{array}{l}\text { Investigação longitudinal } \\
\text { para avaliar o grau de acordo } \\
\text { entre mães e professores, ao } \\
\text { longo de } 3 \text { anos } \\
\text { Analisar o grau de acordo } \\
\text { para os itens } \\
\begin{array}{l}\text { Avaliar problemas } \\
\text { de comportamento e } \\
\text { comportamentos prossociais }\end{array}\end{array}$ & 229 & $\begin{array}{l}\mathrm{T} 1-\text { Kindergarten } \\
\mathrm{T} 2-1^{\circ} \text { Ano } \\
\mathrm{T} 3-2^{\circ} \text { Ano }\end{array}$ & $\begin{array}{l}\text { Maior estabilidade das avaliações das mães no decorrer } \\
\text { dos } 3 \text { anos } \\
\text { Avaliações de professores mais estáveis por períodos de } 2 \\
\text { anos consecutivos (T1-T2) } \\
\text { Maior estabilidade para comportamentos exteriorizados } \\
(r=.28 \text { a } .54) \text { do que interiorizados }(r=.15 \text { a } .37) \text { ou } \\
\text { prossociais }(r=.01 \text { a } .13)\end{array}$ & $\begin{array}{l}\text { 1. Maior grau de acordo pais-professores no } \\
\text { decorrer do pré-escolar do que no } 1^{\circ} \text { ano } \\
\text { 2. Maior grau de acordo pais-professores para } \\
\text { problemas exteriorizados } \\
\text { 3. Menor grau de acordo pais-professores } \\
\text { para problemas interiorizados e para } \\
\text { comportamentos prossociais }\end{array}$ \\
\hline $\begin{array}{l}\text { Gagnon, Vitaro, } \\
\text { \& Tremblay } \\
\text { (1992) }\end{array}$ & $\begin{array}{l}\text { Estudar a influência de } \\
\text { fatores demográficos no } \\
\text { acordo pais-professores } \\
\text { Estudar a prevalência de } \\
\text { cotaçoes de comportamentos } \\
\text { disruptivos }\end{array}$ & 1.924 & $\begin{array}{l}6 \text { anos } \\
\text { (kindergarten) }\end{array}$ & $\begin{array}{l}\text { Correlações de } .26 \text { a } .39 \text { para problemas exteriorizados } \\
\text { (para raparigas e rapazes, respetivamente) } \\
\text { Correlações de } .21 \text { a } .22 \text { para problemas interiorizados } \\
\text { (para raparigas e rapazes, respetivamente) }\end{array}$ & $\begin{array}{l}\text { 1. Reduzido a moderado grau de acordo entre } \\
\text { informadores } \\
\text { 2. Apesar da semelhança na prevalência } \\
\text { dos comportamentos, existem algumas } \\
\text { discrepâncias mais flagrantes entre os } \\
\text { informadores (e.g., item "Desobediente" com } \\
\text { uma prevalência de } 74.5 \% \text { de acordo com as } \\
\text { mães contra } 19.4 \% \text { para os professores) }\end{array}$ \\
\hline $\begin{array}{l}\text { Hinshaw, Han, } \\
\text { Erhardt, \& Huber } \\
\text { (1992) }\end{array}$ & $\begin{array}{l}\text { Estudar a relação entre } \\
\text { as cotações de pais, } \\
\text { professores e a observação } \\
\text { de comportamentos sociais } \\
\text { numa situação de jogo, } \\
\text { numa amostra composta } \\
\text { por } 3 \text { grupos de crianças: } \\
\text { grupo de comparaçã, } \\
\text { grupo exteriorizado e grupo } \\
\text { interiorizado }\end{array}$ & 45 & 3 aos 6 anos & $\begin{array}{l}\text { Correlações pais-professores de } .13(n s) \text { para problemas } \\
\text { interiorizados e de } .32 \text { para problemas exteriorizados } \\
\text { Cotações dos pais referentes a problemas interiorizados } \\
\text { tendem a predizer observações de comportamentos de } \\
\text { isolamento }(r=.28) \\
\text { Cotações de professores estão associadas à observação de } \\
\text { comportamentos agressivos }(r=.52)\end{array}$ & $\begin{array}{l}\text { 1. Correlações mais baixas para os problemas } \\
\text { interiorizados } \\
\text { 2. Validade diferente dos relatos de pais e } \\
\text { professores utilizando índices de observação de } \\
\text { comportamentos interiorizados e exteriorizados } \\
\text { como critério } \\
\text { 3. Necessidade dos investigadores que } \\
\text { trabalhem com a população pré-escolar } \\
\text { selecionarem os informadores atendendo à } \\
\text { informação única que podem facultar para } \\
\text { determinados domínios comportamentais }\end{array}$ \\
\hline $\begin{array}{l}\text { Culp, Howell, } \\
\text { Culp, \& } \\
\text { Blankemeyer } \\
(2001)\end{array}$ & $\begin{array}{l}\text { Averiguar até que ponto pais } \\
\text { e professores "veem a mesma } \\
\text { coisa" (título do artigo), } \\
\text { na avaliação de problemas } \\
\text { de comportamento numa } \\
\text { amostra de crianças vítimas } \\
\text { de maus-tratos }\end{array}$ & 33 & $\begin{array}{l}\text { Média de idades: } \\
4 \text { anos }\end{array}$ & $\begin{array}{l}\text { Correlações de -.14 a .09 } \\
\text { Resultados médios para as várias subescalas, mais } \\
\text { elevados de acordo com as cotações dos pais, com exceção } \\
\text { da impulsividade, ligeiramente superior nas cotações de } \\
\text { professores }\end{array}$ & $\begin{array}{l}\text { 1. Pais e professores encaram a criança de } \\
\text { forma diferente } \\
\text { 2. Mais do que analisar quem é o melhor } \\
\text { informador ou que tipo de comportamento } \\
\text { necessita de intervenção, é imperativo } \\
\text { desenvolver medidas adequadas para a } \\
\text { identificação de problemas exteriorizados ou } \\
\text { interiorizados, com vista a uma intervenção } \\
\text { precoce }\end{array}$ \\
\hline Goodman (2001) & $\begin{array}{l}\text { Estudar o grau de acordo } \\
\text { pais-professores na avaliação } \\
\text { de comportamentos } \\
\text { prossociais e problemas de } \\
\text { comportamento }\end{array}$ & 7.313 & 5 aos 15 anos & $\begin{array}{l}\text { Correlações de } .25 \text { a } .48 \\
\text { Correlações mais elevadas para o resultado total e escala } \\
\text { de Hiperatividade-Desatenção }\end{array}$ & $\begin{array}{l}\text { 1. Correlações moderadas entre os } \\
\text { informadores, mas superiores ao valor de } \\
\text { referência da meta-análise de Achenbach et al. } \\
\text { (1987), igual a . } 27 \text {. }\end{array}$ \\
\hline $\begin{array}{l}\text { Winsler \& } \\
\text { Wallace (2002) }\end{array}$ & 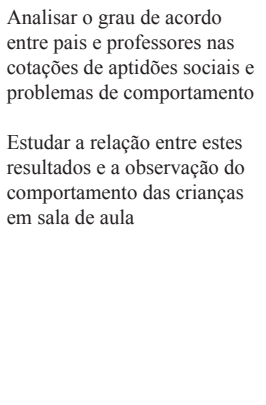 & 47 & 3 aos 4 anos & $\begin{array}{l}\text { Grau de acordo pais-professores modesto (-.09 a .38), } \\
\text { sendo mais reduzido para as aptidões sociais }(-.09 \text { a } .27) \\
\text { Grau de acordo pais-professores modesto, quer para } \\
\text { os problemas interiorizados }(.15 \text { a } .36) \text {, quer para os } \\
\text { problemas de caráter exteriorizado }(.29 \text { a } .38) \\
\text { Para o acordo entre informadores em função da idade da } \\
\text { criança encontra-se uma maior amplitude de resultados } \\
\text { para os } 3 \text { anos (-.17 a .68) em comparação com os } 4 \text { anos } \\
\text { (-.02 a .45) } \\
\text { Maior relação entre observação em sala de aula de } \\
\text { comportamentos de afiliação social com os pares e } \\
\text { cotações de aptidões sociais feitas por professores }(r=.40) \\
\text { do que por pais }(r=.14)\end{array}$ & $\begin{array}{l}\text { 1. Maior grau de acordo entre informadores } \\
\text { no que diz respeito aos problemas de } \\
\text { comportamento em comparação com as } \\
\text { aptidões sociais } \\
\text { 2. Os pais tendem a cotar os seus filhos com } \\
\text { mais problemas que os professores } \\
\text { 3. Em comparação com as avaliações dos pais, } \\
\text { as cotações de aptidões sociais e problemas } \\
\text { de comportamento feitas pelos professores } \\
\text { apresentam um maior grau de associação com } \\
\text { a observação do comportamento das crianças } \\
\text { em sala de aula }\end{array}$ \\
\hline
\end{tabular}


Tabela 1. (continuação) Sumários dos Estudos Revistos: Acordo entre Informadores dos Contextos Familiar e Escolar

\begin{tabular}{|c|c|c|c|c|c|}
\hline $\begin{array}{l}\text { Autores } \\
\text { Ano }\end{array}$ & Objetivos & $\begin{array}{c}N \\
\text { Amostra }\end{array}$ & $\begin{array}{c}\text { Idade ou } \\
\text { Escolaridade }\end{array}$ & Principais Resultados & Conclusões \\
\hline $\begin{array}{l}\text { Gross, Fogg, } \\
\text { Garvey, \& Julion } \\
\text { (2004) }\end{array}$ & $\begin{array}{l}\text { Analisar o acordo pais- } \\
\text { professores relativamente a } \\
\text { problemas de comportamento } \\
\text { em crianças provenientes de } \\
\text { baixo nível socioeconómico }\end{array}$ & 241 & 2 aos 4 anos & $\begin{array}{l}\text { Correlações baixas entre informadores }(r=.17) \\
\text { Uma percentagem reduzida de crianças recebe cotações } \\
\text { elevadas nos problemas de comportamento por parte de } \\
\text { ambos os informadores (pais e professores) }(2.5 \%)\end{array}$ & $\begin{array}{l}\text { 1. Os pais cotam as crianças com } \\
\text { mais problemas de comportamento } \\
\text { comparativamente aos professores } \\
\text { 2. Importância de recolher a perspetiva de } \\
\text { vários informadores, o mais precocemente } \\
\text { possível }\end{array}$ \\
\hline $\begin{array}{l}\text { Renk \& Phares } \\
\text { (2004) }\end{array}$ & $\begin{array}{l}\text { Estudo de meta-análise } \\
\text { (Final dos anos } 80 \text { a final dos } \\
\text { anos 90) } \\
\text { Estudar o acordo entre } \\
\text { informadores nas cotações da } \\
\text { competência social }\end{array}$ & 74 estudos & $\begin{array}{l}\text { Pré-escolar ao } \\
\text { ensino secundário }\end{array}$ & $\begin{array}{l}\text { Correlação média para o acordo pais-professores }=.43 \\
\text { Correlação média ponderada para o acordo pais- } \\
\text { professores }=.38 \\
\text { Maior grau de acordo pais-professores para medidas } \\
\text { sociométricas }(.73) \text { face a escalas de avaliação }(.36)\end{array}$ & $\begin{array}{l}\text { 1. Importância de incluir a perspetiva de vários } \\
\text { informadores ao avaliar a competência social de } \\
\text { crianças e adolescentes } \\
2 \text {. Necessidade dos profissionais terem em } \\
\text { conta a competência social das crianças nos } \\
\text { seus procedimentos de avaliação/intervenção, } \\
\text { mais do que manterem o foco nas dificuldades } \\
\text { relatadas pelas famílias }\end{array}$ \\
\hline $\begin{array}{l}\text { Tarren-Sweeney, } \\
\text { Hazell, \& Carr } \\
(2004)\end{array}$ & $\begin{array}{l}\text { Analisar o grau de acordo } \\
\text { entre pais/famílias de } \\
\text { acolhimento e professores } \\
\text { em relação a problemas de } \\
\text { comportamento }\end{array}$ & 47 & 5 aos 11 anos & $\begin{array}{l}\text { Correlações entre as cotações dos dois informadores } \\
\text { para o total dos problemas de comportamento }(r=.71) \mathrm{e} \\
\text { problemas exteriorizados }(r=.78) \text { superiores aos valores } \\
\text { apontados na literatura } \\
\text { Correlação para os problemas de tipo interiorizado }(\mathrm{r}= \\
.23) \text { comparável aos estudos de referência }\end{array}$ & $\begin{array}{l}\text { 1. Os resultados obtidos permitem questionar o } \\
\text { que terá contribuído para a discrepância entre } \\
\text { este estudo com pais/famílias de acolhimento } \\
\text { (grau de acordo elevado para o resultado } \\
\text { total e problemas exteriorizados) e os valores } \\
\text { reportados na literatura } \\
\text { 2. Maior grau de acordo justificado como } \\
\text { possível resultado de um maior conhecimento } \\
\text { e compreensão da criança que têm ao seu } \\
\text { cuidado, por parte das famílias de acolhimento }\end{array}$ \\
\hline $\begin{array}{l}\text { Herrera \& Little } \\
(2005)\end{array}$ & $\begin{array}{l}\text { Estudar o grau de acordo } \\
\text { entre pais e professores em } \\
\text { duas amostras, normal e } \\
\text { clínica, quanto a problemas } \\
\text { de comportamento } \\
\text { exteriorizados }\end{array}$ & $\begin{array}{l}22 \\
\text { (Amostra } \\
\text { normal) } \\
11 \\
\text { (Amostra } \\
\text { clínica) }\end{array}$ & 3 aos 5 anos & $\begin{array}{l}\text { Amostra normal: Correlação de } .10 \text { (coeficiente intraclasse } \\
=.04 \text { ) para o grau de acordo pais-professores para os } \\
\text { problemas de comportamento } \\
\text { Amostra clínica: Correlação de } .91 \text { (coeficiente intraclasse } \\
=.89 \text { ) para o grau de acordo pais-professores para os } \\
\text { problemas de comportamento }\end{array}$ & $\begin{array}{l}\text { 1. Os pais apontam níveis mais elevados de } \\
\text { problemas de comportamento aos seus filhos do } \\
\text { que os professores (em ambas as amostras) } \\
\text { 2. Maior grau de acordo pais-professores na } \\
\text { amostra clínica }\end{array}$ \\
\hline $\begin{array}{l}\text { Gagnon, Nagle, } \\
\& \text { Nickerson } \\
(2007)\end{array}$ & $\begin{array}{l}\text { Estudar o grau de acordo e } \\
\text { consistência entre avaliações } \\
\text { de pais-professores ao } \\
\text { nível do jogo entre pares } \\
\text { e do desenvolvimento } \\
\text { socioemocional }\end{array}$ & 83 & 4 anos & $\begin{array}{l}\text { Correlações pais-professores de }-.19 \text { a } .18 \text { para o jogo } \\
\text { entre pares } \\
\text { Correlações pais-professores de } .13 \text { a } .46 \text { para a avaliação } \\
\text { do desenvolvimento socioemocional }\end{array}$ & $\begin{array}{l}\text { 1. Pais cotam o comportamento de jogo como } \\
\text { mais disruptivo e menos interativo do que } \\
\text { professores } \\
\text { 2. Ao utilizar escalas de avaliação do } \\
\text { comportamento, o avaliador deverá antecipar } \\
\text { a obtenção de um reduzido grau de acordo e } \\
\text { considerar as cotações de cada informador como } \\
\text { contributos individuais para a avaliação }\end{array}$ \\
\hline $\begin{array}{l}\text { Lane, Stanton- } \\
\text { Chapman, } \\
\text { Jamison, \& } \\
\text { Phillips (2007) }\end{array}$ & $\begin{array}{l}\text { Analisar as expectativas de } \\
\text { pais e professores acerca das } \\
\text { aptidões sociais necessárias } \\
\text { para o sucesso em contexto } \\
\text { escolar } \\
\text { Analisar a frequência com } \\
\text { que dois informadores cotam } \\
\text { determinadas aptidões sociais } \\
\text { como críticas para o sucesso } \\
\text { escolar }\end{array}$ & 124 & 2 aos 6 anos & $\begin{array}{l}\text { Itens apontados pelos professores como críticos para o } \\
\text { sucesso: "Segue as nossas instruções" }(80 \%) \text {, "Controla } \\
\text { o temperamento em situações conflituosas com adultos" } \\
(77 \%) \text { e "Controla o temperamento em situações } \\
\text { conflituosas com os pares" ( } 63 \%) \\
\text { Itens apontados pelos pais (para além dos três itens } \\
\text { anteriores): "Cumpre as regras da casa" ( } 77 \% \text { ) e "Fala } \\
\text { num tom de voz apropriado em casa" }(73 \%)\end{array}$ & $\begin{array}{l}\text { 1. Ambos os informadores colocam um } \\
\text { forte peso em aptidões de cooperação social, } \\
\text { contrariamente aos domínios da assertividade e } \\
\text { autocontrolo, mais valorizados pelos pais } \\
\text { 2. Ênfase na importância da compreensão das } \\
\text { diferentes expectativas, de forma a facilitar } \\
\text { a comunicação e colaboração entre pais e } \\
\text { professores, para facultar às crianças uma } \\
\text { experiência educacional sólida }\end{array}$ \\
\hline $\begin{array}{l}\text { Murray et al. } \\
(2007)\end{array}$ & $\begin{array}{l}\text { Analisar o grau de acordo } \\
\text { entre pais e professores } \\
\text { na cotação de sintomas } \\
\text { de défice de atenção/ } \\
\text { perturbação de hiperatividade } \\
\text { em criaças pré-escolares } \\
\text { referenciadas para um estudo } \\
\text { de tratamento na Perturbação } \\
\text { de Hiperatividade e Défice de } \\
\text { Atenção (PHDA) }\end{array}$ & 452 & 3 aos 5 anos & $\begin{array}{l}\text { Correlação pais-professores de } .24 \text { para comportamentos } \\
\text { de desatenção } \\
\text { Correlação pais-professores de } .26 \text { para comportamentos } \\
\text { de hiperatividade } \\
\text { Correlações pais-professores de } .01 \text { a } .28 \text { para sintomas } \\
\text { considerados individualmente }\end{array}$ & $\begin{array}{l}\text { 1. As crianças pré-escolares com sintomas de } \\
\text { PHDA apresentam comportamentos distintos } \\
\text { em diferentes contextos } \\
\text { 2. A recolha de informação atendendo à } \\
\text { perspetiva de múltiplos informadores revela-se } \\
\text { crítica na obtenção de um retrato mais completo } \\
\text { do reportório comportamental das crianças } \\
\text { pré-escolares }\end{array}$ \\
\hline $\begin{array}{l}\text { Miner \& Clarke- } \\
\text { Stewart (2008) }\end{array}$ & $\begin{array}{l}\text { Analisar a trajetória dos } \\
\text { problemas de comportamento } \\
\text { exteriorizados, avaliados por } \\
\text { pais e professores dos } 2 \text { aos } 9 \\
\text { anos de idade }\end{array}$ & 1.364 & $\begin{array}{l}2 \text { a } 4 \text { anos; } \\
7 \text { e } 9 \text { anos }\end{array}$ & $\begin{array}{l}\text { Grau de acordo reduzido a moderadamente reduzido entre } \\
\text { informadores, de } .19 \text { a } .34(\mathrm{M}=.25)\end{array}$ & $\begin{array}{l}\text { 1. As mães tendem, sistematicamente, a } \\
\text { cotar os seus filhos com mais problemas } \\
\text { de comportamento exteriorizados face aos } \\
\text { informadores em contexto escolar }\end{array}$ \\
\hline $\begin{array}{l}\text { Stefan, Balaj, } \\
\text { Porumb, Albu, \& } \\
\text { Miclea (2009) }\end{array}$ & $\begin{array}{l}\text { Desenvolver ferramentas } \\
\text { de screening para a } \\
\text { avaliação de competências } \\
\text { sociais e emocionais, } \\
\text { desenvolvimentalmente } \\
\text { adequadas à idade pré- } \\
\text { escolar } \\
\text { Analisar o grau de acordo } \\
\text { entre pais e professores }\end{array}$ & 824 & 2.5 aos 7.5 anos & $\begin{array}{l}\text { Grau de acordo mais elevado para os } 4-5 \text { anos }(r= \\
.36) \text { face aos } 2.5-4 \text { anos e } 5-7.5 \text { anos }(r=.15 \text { e } .14, \\
\text { respetivamente) }\end{array}$ & $\begin{array}{l}\text { 1. Grau de acordo reduzido entre pais } \\
\text { e professores ao nível da avaliação de } \\
\text { competências sociais e emocionais } \\
\text { 2. A incongruência dos comportamentos das } \\
\text { crianças em casa e na escola aponta para a } \\
\text { necessidade de uma abordagem com recurso } \\
\text { a múltiplos informadores e vários métodos de } \\
\text { recolha de dados }\end{array}$ \\
\hline
\end{tabular}


Tabela 1. (continuação) Sumários dos Estudos Revistos: Acordo entre Informadores dos Contextos Familiar e Escolar

\begin{tabular}{|c|c|c|c|c|c|}
\hline $\begin{array}{c}\text { Autores } \\
\text { Ano }\end{array}$ & Objetivos & $\begin{array}{c}N \\
\text { Amostra }\end{array}$ & $\begin{array}{c}\text { Idade ou } \\
\text { Escolaridade }\end{array}$ & Principais Resultados & Conclusões \\
\hline $\begin{array}{l}\text { Phillips \& } \\
\text { Lonigan (2010) }\end{array}$ & 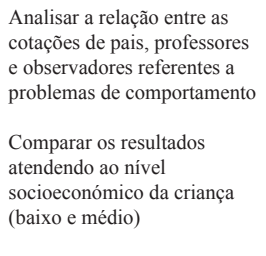 & 365 & 3 e 4 anos & $\begin{array}{l}\text { Correlações pais-professores de } .15 \text { a } .24 \text { no grupo de } \\
\text { crianças de nível socioeconómico baixo } \\
\text { Correlações pais-professores de } .24 .52 \text { no grupo de } \\
\text { crianças de nível socioeconómico médio }\end{array}$ & $\begin{array}{l}\text { 1. Grau de acordo pais-professores mais } \\
\text { reduzido para crianças de nível socioeconómico } \\
\text { mais baixo } \\
\text { 2. Pais e professores tendem a cotar as crianças } \\
\text { de níveis socioeconómicos mais baixos com } \\
\text { mais problemas de comportamento e menos } \\
\text { comportamentos prossociais } \\
\text { 3. Necessidade de recorrer a múltiplas fontes } \\
\text { de informação na avaliação de problemas } \\
\text { socioemocionais das crianças }\end{array}$ \\
\hline $\begin{array}{l}\text { Stone, Otten., } \\
\text { Engels, Vermulst, } \\
\text { \& Janssens } \\
(2010)\end{array}$ & $\begin{array}{l}\text { Revisão das propriedades } \\
\text { psicométricas de um } \\
\text { instrumento de avaliação } \\
\text { com versão para pais e } \\
\text { professores } \\
\text { Resumir oito estudos de } \\
\text { acordo entre pais-professores } \\
\text { realizados entre } 1997 \text { e } 2008\end{array}$ & 14.811 & 4 aos 12 anos & $\begin{array}{l}\text { Grau de acordo pais-professores entre } .26 \\
\text { (comportamentos prossociais) a } .47 \text { (comportamentos de } \\
\text { hiperatividade/desatenção) } \\
\text { Menor grau de acordo para comportamentos prossociais e } \\
\text { interiorizados face aos exteriorizados }\end{array}$ & $\begin{array}{l}\text { 1. Grau de acordo modesto entre cotações de } \\
\text { pais e professores, mas superiores ao valor de } \\
\text { referência da meta-análise de Achenbach et } \\
\text { al. (1987), com exceção dos comportamentos } \\
\text { prossociais. }\end{array}$ \\
\hline $\begin{array}{l}\text { Cranes, Minsic, \& } \\
\text { Winsler (2011) }\end{array}$ & $\begin{array}{l}\text { Analisar o grau de acordo na } \\
\text { competência socioemocional } \\
\text { de crianças provenientes } \\
\text { de níveis socioeconómicos } \\
\text { baixos, avaliada por pais e } \\
\text { professores }\end{array}$ & 7.756 & 3 e 4 anos & $\begin{array}{l}\text { Grau de acordo reduzido a moderado entre informadores } \\
(r=.20 \mathrm{a} .28)\end{array}$ & $\begin{array}{l}\text { 1. Grau de acordo modesto e congruente com os } \\
\text { estudos de referência (Achenbach et al., 1987) } \\
\text { 2. Pais cotam os seus filhos tanto com mais } \\
\text { comportamentos positivos como com mais } \\
\text { problemas de comportamento }\end{array}$ \\
\hline $\begin{array}{l}\text { Klyce, Conger, } \\
\text { Conger, \& Dumas } \\
\text { (2011) }\end{array}$ & $\begin{array}{l}\text { Estudo longitudinal } \\
\text { para analisar o grau de } \\
\text { acordo na competência e } \\
\text { disfuncionamento de crianças } \\
\text { pré-escolares, avaliadas por } \\
\text { pais e respetivos professores }\end{array}$ & 610 & $\begin{array}{l}\text { Média de } 4.4 \text { anos } \\
\quad(\mathrm{DP}=0.78)\end{array}$ & $\begin{array}{l}\text { Grau de acordo entre informadores de } .18 \text { (comportamento } \\
\text { agressivo; narcisismo) a } .22 \text { (comportamento afetivo) }\end{array}$ & $\begin{array}{l}\text { 1. Reduzido grau de acordo pais-professores } \\
\text { 2. Os resultados sugerem que a competência e } \\
\text { o disfuncionamento podem desenvolver-se em } \\
\text { resposta aos diferentes requisitos dos contextos } \\
\text { de interação da criança (familiar e escolar) } \\
\text { 3. Importância de incluir a avaliação de } \\
\text { comportamentos prossociais, exteriorizados e } \\
\text { interiorizados na prática clínica }\end{array}$ \\
\hline $\begin{array}{l}\text { Liu, Cheng, \& } \\
\text { Leung (2011) }\end{array}$ & $\begin{array}{l}\text { Analisar o grau de } \\
\text { acordo nos problemas de } \\
\text { comportamento avaliados por } \\
\text { pais e professores }\end{array}$ & 876 & 50 a 71 meses & $\begin{array}{l}\text { Grau de acordo reduzido entre pais e professores, com } \\
\text { uma correlação média de } .18 \text { (amplitude de } .04 \text { a .25) }\end{array}$ & $\begin{array}{l}\text { 1. Acordo reduzido entre as cotações de pais e } \\
\text { professores } \\
\text { 2. Maior grau de acordo entre informadores } \\
\text { para os problemas exteriorizados }\end{array}$ \\
\hline
\end{tabular}

Vitaro et al., 1991; Winsler \& Wallace, 2002). Acresce, ainda, a existência de um maior grau de acordo entre informadores para problemas de comportamento do que para aptidões sociais (Goodman, 2001; Stone et al., 2010; Vitaro et al., 1991; Winsler \& Wallace, 2002). Atendendo à variável idade existem dados contraditórios, com alguns estudos a apontar um maior grau de acordo no pré-escolar (Vitaro et al., 1991) e outros a remeter essa maior concordância para as informações prestadas sobre crianças mais velhas (Verhulst \& Akkerhuis, 1989). Finalmente, no que diz respeito a problemas mais específicos, quer interiorizados (e.g., ansiedade), quer exteriorizados (e.g., agressão), observam-se níveis reduzidos a moderados de acordo (Stone et al., 2010; Vitaro et al., 1991).

\section{Discussão}

Uma primeira inferência que é possível fazer a partir dos estudos analisados indica que, apesar do aumento do interesse pelos comportamentos positivos das crianças (e.g., aptidões sociais), existe ainda uma primazia de estudos realizados acerca do acordo entre informadores exclusivamente focados em problemas de comportamento (11 dos 22 estudos) em comparação com os estudos cujo foco são as competências das crianças (somente 3 dos 22 estudos são centrados apenas em aptidões sociais). Por outro lado, é notória nesta revisão a referida menor participação paterna ao nível da investigação, face à maior disponibilidade por parte das mães (Phares \& Compas, 1992; Phares et al., 2005).
Os 22 estudos revistos indicam que, de uma forma geral, apesar de pais e professores poderem facultar informação, em primeira mão, acerca dos problemas socioemocionais da criança (Hinshaw et al., 1992), o grau de acordo encontrado entre estes informadores em escalas de avaliação do comportamento da criança tem sido reduzido a moderado, em amostras com características diversas (e.g., amostras clínicas, população geral), ou considerando diferentes tipos de comportamentos (e.g., problemas de comportamento específicos, aptidões sociais) (e.g., Achenbach et al., 1987; Cranes et al., 2011; Klyce et al., 2011; Renk \& Phares, 2004; Verhulst \& Akkerhuis, 1989; Winsler \& Wallace, 2002). Uma justificação plausível para este padrão de resultados relaciona-se com a diferença de contextos em que pais e professores interagem com as crianças, o que poderá levar a perspetivas divergentes acerca do comportamento destas. Para tal contribuem os tipos diferentes de atividades desenvolvidas, as pessoas com quem as crianças interagem nos diferentes contextos, as diferentes exigências em termos de comportamento prossocial e até mesmo a própria expectativa e base de referência do comportamento normativo pela qual os informadores regem a sua avaliação (Major, 2011).

Acresce que os resultados da revisão da literatura indicam uma maior propensão dos pais para cotarem os seus filhos com mais problemas de comportamento, comparativamente a informadores do contexto escolar, quer seja pelo maior tempo passado com a criança, pela observação desta num leque mais diversificado de situações, menor tolerância para problemas 
considerados perturbadores, ou expectativas irrealistas sobre o que é o comportamento normativo em idade pré-escolar (e.g., Cranes et al., 2011; Gagnon et al., 1992; Gross et al., 2004; Herrera \& Little, 2005; Verhulst \& Akkerhuis, 1989; Winsler \& Wallace, 2002). Esta verificação remete para a importância e a necessidade de educação parental no sentido de aumentar a informação sobre o que é normativo em cada idade e, desse modo, ajustar as expectativas/respostas dos pais face aos comportamentos dos seus filhos.

O maior grau de acordo para comportamentos de tipo exteriorizado pode ser justificado pela maior visibilidade e incómodo causados por este tipo de problemas (e.g., Achenbach et al., 1987; Stone et al., 2010; Vitaro et al., 1991; Winsler \& Wallace, 2002). Por outro lado, estes resultados poderão igualmente chamar a atenção para o perigo da não identificação/negligência de problemas interiorizados por parte de alguns dos adultos que lidam com a criança, podendo levar à ausência de procura de apoio especializado, em casos em que tal poderia ser justificado. Também as competências sociais das crianças são alvo de menor acordo por parte dos adultos que interagem com elas (Stone et al., 2010), o que poderá refletir alguma dificuldade na perceção e identificação destes comportamentos por parte dos informadores ou, ainda, ser o reflexo da diferença de aptidões sociais que os informadores podem observar, atendendo ao seu contexto de interacção com a criança (Achenbach et al., 1987; Goodman, 2001) e aos requisitos sociais diversos entre o contexto familiar e o escolar.

No que diz respeito à variável idade, verifica-se a existência de inconsistências entre os resultados dos estudos, com alguns deles a apontarem um maior grau de acordo no pré-escolar (Vitaro et al., 1991), o que se pode justificar pelo ambiente mais relaxado do jardim-de-infância, semelhante ao contexto familiar, enquanto outros encontram níveis mais elevados de acordo em crianças mais velhas (Verhulst \& Akkerhuis, 1989). No geral, estes resultados reforçam a obtenção de um reduzido a moderado grau de concordância entre informadores, independentemente da idade das crianças, apontando a necessidade de mais estudos longitudinais.

As correlações reduzidas a moderadas obtidas nos estudos de acordo entre informadores não devem, contudo, ser assumidas como sinónimo de falta de fiabilidade das escalas de avaliação (Hundert et al., 1997), ou como resultantes do facto de uma das fontes de informação ser pouco fiável/ válida. Assim, ao mesmo tempo que se reconhece que cada informador pode contribuir com informação válida, também se torna evidente que a informação disponibilizada por cada fonte corrobora apenas parcialmente a proveniente de outras fontes (Achenbach \& McConaughy, 1987; Achenbach et al., 1987; Gagnon et al., 1992). Por conseguinte, ao utilizar escalas de avaliação do comportamento o examinador deverá antecipar reduzidos níveis de acordo e optar por considerar as cotações de cada informador como contributos individuais para a avaliação e oportunidades para aumentar a informação obtida e para estabelecer inferências acerca do funcionamento familiar e da própria criança (Gagnon et al., 2007; Kamphaus \& Frick, 1996).

Neste contexto, a revisão da literatura efetuada, ao colocar em destaque o reduzido a moderado grau de acordo entre informadores, torna recomendável prudência por parte de clínicos e investigadores na leitura dos dados recolhidos a partir de adultos diferentes, não assumindo que a informação facultada por um informador particular possa ser substituída pela de outro (Stranger \& Lewis, 1993). Cada informador oferece uma contribuição única que amplifica a precisão no processo de avaliação em curso (Grietens et al., 2004). Neste sentido, apesar de pais e professores serem considerados como informadores essenciais na compreensão do funcionamento socioemocional das crianças pré-escolares, a existência de desacordo entre eles quanto ao comportamento da criança pode ser encarada como uma vantagem, ao chamar a atenção para o facto de que um dos contextos pode estar a ser mais efetivo em lidar com as características da criança do que o outro. Por exemplo, a identificação de um problema comportamental ou duma aptidão social específica num contexto mas não no outro poderão fornecer pistas importantes quanto às contingências ambientais e ao modo de lidar com a criança que melhor contribuem para a otimização das suas potencialidades. Esta estratégia de integração da informação e o desenvolvimento de parcerias casa-escola colocará pais e professores numa posição mais favorável quanto à prevenção da escalada de problemas de comportamento, antes de a criança transitar para a escolaridade formal (Gagnon et al., 2007; Herrera \& Little, 2005; Renk, 2005).

Dado não existir um padrão ideal ("golden standard") acerca de quem é o melhor informador sobre o funcionamento da criança (Kroes, 2006), o objetivo principal será a obtenção de uma maior quantidade de informação, segundo diversos pontos de vista, para alcançar uma descrição e compreensão válidas e exaustivas dos problemas apresentados pelas crianças. Por conseguinte, e respondendo à questão inicial - pais e/ou professores? -, a literatura permite concluir que o recurso a ambos será fundamental para tomar decisões sobre a necessidade ou não de um plano interventivo, que tipo de intervenção se poderá revelar mais apropriado e que comportamentos deverão ser alvo de intervenção (Culp et al., 2001).

\section{Implicações para estudos futuros}

A revisão da literatura efetuada permitiu destacar alguns aspetos relativamente consensuais, ao longo de mais de 20 anos de investigação, no que toca à comparação entre as informações provenientes de várias fontes, tais como a obtenção de informação diversa por parte de diferentes informadores, o facto de os pais tendencialmente apontarem mais problemas às crianças do que os professores e, ainda, a existência de um maior grau de acordo para problemas exteriorizados do que interiorizados. Cabe ao profissional considerar e compreender o sentido da perspetiva única de cada um dos informadores, aliada às características particulares de cada criança e contexto, com o objetivo de obter um retrato o mais fidedigno e válido possível do respetivo padrão comportamental.

Uma vez que pais e professores mantêm diferentes relações com a criança e a observam em condições diversas, as discrepâncias entre os seus relatos não representam uma surpresa ou uma novidade (Klyce et al., 2011; Seabra-Santos 
\& Gaspar, 2012). Neste sentido, para além do estudo do acordo entre informadores, a literatura especializada aponta a análise das discrepâncias entre a informação facultada por várias fontes como uma área a explorar (Crane et al., 2011; De Los Reyes \& Kazdin, 2005), uma vez que a investigação recentemente desenvolvida tem sugerido que as discrepâncias entre informadores poderão ter subjacentes características particulares de pais, crianças ou do funcionamento familiar, as quais importa esclarecer (Berg-Nielsen, 2012; De Los Reyes \& Kazdin, 2005; Phillips et al., 2010). Por conseguinte, nos estudos sobre o acordo entre informadores quanto ao funcionamento socioemocional das crianças deverá ser ponderado o impacto de variáveis relativas às características da criança (e.g., idade, sexo), dos pais/informadores (e.g., presença de depressão) e da família (e.g., mal-estar familiar) (Berg-Nielsen, 2012; De Los Reyes \& Kazdin, 2005), assim como ao método utilizado para recolher a informação (Renk, 2005). A título de exemplo, a verificação de que mães deprimidas tendem a cotar os seus filhos com mais problemas de comportamento pode justificar o recurso a informadores-critério (criterion raters) considerados mais objetivos, como estratégia de validação (ou não) dos relatos maternos (Ordway, 2011). Estes informadores poderão ser outros cuidadores ou professores da criança.

Também o recurso a métodos estatísticos mais sofisticados, tais como Modelos de Equações Estruturais (SEM), como forma de facultar informação mais detalhada acerca da influência das características dos informadores nos resultados obtidos (Ordway, 2011), poderá revelar-se uma mais-valia e um complemento aos estudos de acordo entre informadores sustentados em análises correlacionais ou de diferenças de médias (como é o caso da maior parte dos estudos apresentados nesta revisão). A aplicação do Attribution Bias Context Model, proposto por De Los Reyes e Kazdin (2005), também se apresenta como uma alternativa a explorar na identificação de discrepâncias entre pares de informadores (e.g., pais-professores), ao ter em consideração as atribuições, perspetivas e interações entre informadores, atendendo aos objetivos do processo de avaliação clínica.

Quanto às propriedades psicométricas dos instrumentos utilizados nos 22 estudos analisados, destaca-se o recurso a escalas de avaliação devidamente validadas (e.g., TRF; PKBS-2). No entanto, uma vez que, tal como foi referido para os informadores, também para as escalas de avaliação não existe um "golden standard", sublinhamos a vantagem do uso de escalas com o mesmo conjunto de itens a serem cotados por pais e por professores (e.g., PKBS-2), como forma de reduzir a variância associada ao instrumento (Stranger \& Lewis, 1993). O estudo do acordo entre informadores ao nível dos itens, não se focando meramente nos resultados de escalas/subescalas, merece também ser apontado como uma proposta para estudos futuros.

Destaca-se, igualmente, a necessidade de mais investigação acerca do acordo em relação às competências/ comportamentos positivos das crianças (e.g., aptidões sociais), as quais têm ocupado um papel secundário em comparação com os problemas de comportamento (Renk, 2005).

A tendência ancestral da maior disponibilidade por parte da figura materna para se dedicar aos filhos e a tudo o que com eles se relaciona é espelhada na taxa significativamente superior de respostas de mães, comparativamente às de pais, nos estudos analisados (Phares \& Compas, 1992). Por outro lado, o aumento do envolvimento dos progenitores masculinos na educação dos seus filhos que se tem vindo a verificar nos últimos anos (Phares et al., 2005), recomenda que maior atenção seja dada à perspetiva paterna. Nesse sentido, deveria ser feito um maior esforço, ao nível da investigação, com vista a fomentar a participação dos paishomens em estudos deste tipo. Um modo de alcançar este objetivo poderá ser, por exemplo, solicitando diretamente que sejam os pais-homens (só ou também) a responder aos questionários.

De toda esta abordagem fica claro que, mais de 20 anos após a meta-análise de Achenbach et al. (1987), prevalece a ideia de que é importante recolher um leque amplo de informação acerca do funcionamento da criança em diferentes situações e segundo a perspetiva de vários informadores. Por outro lado, não existindo um informadorchave para a obtenção de um retrato válido e fidedigno do perfil comportamental da criança pré-escolar, a opção de recolher a perspetiva de pais e professores revela-se uma mais-valia, não apenas em situações que envolvam a identificação/avaliação de crianças em risco de desenvolver problemas de comportamento, mas também, em geral, com o objetivo de fomentar um desenvolvimento o mais harmonioso possível da criança, nos seus vários contextos. Neste sentido, é fundamental uma boa comunicação entre os diversos adultos que lidam com a criança, a qual pode ser conseguida através de um maior envolvimento dos pais nas atividades do jardim-de-infância e um maior acompanhamento por parte destes do desenvolvimento, não só académico, mas também socioemocional das crianças (e.g., relação com pares e com adultos), considerado como um patamar para o bem-estar futuro.

\section{Referências}

Achenbach, T. M. (1991). Manual for the Teacher's Report Form and 1991 Profile. Burlington, VT: University of Vermont, Department of Psychiatry.

Achenbach, T. M., \& McConaughy, S. H. (1987). Empirically based assessment of child and adolescent psychopathology: Practical applications. Developmental Clinical Psychology and Psychiatry (Vol. 13). Newbury Park, CA: Sage.

Achenbach, T. M., McConaughy, S. H., \& Howell, C. T. (1987). Child/adolescent behavioral and emotional problems: Implications of cross-informant correlations for situational specificity. Psychological Bulletin, 101(2), 213-232.

Berg-Nielsen, T. S., Solheim, E., Belsky, J., \& Wichstrom, J. (2012). Preschoolers' psychosocial problems: In the eyes of the beholder? Adding teacher characteristics as determinants of discrepant parent-teacher reports. Child Psychiatry and Human Development, 43, 393-413. doi: 10.1007/s10578-011-0271-0 
Crane, J., Mincic, M. S., \& Winsler, A. (2011). Parentteacher agreement and reliability on the Devereux Early Childhood Assessment (DECA) in English and Spanish for ethnically diverse children living in poverty. Early Education and Development, 22(3), 520-547. doi: 10.1080/10409289.2011.565722

Culp, R. E., Howell, C. S., Culp, A. M., \& Blankemeyer, M. (2001). Maltreated children's emotional and behavioral problems: Do teachers and parents see the same things? Journal of Child and Family Studies, 10(1), 39-50.

De Los Reyes, A., \& Kazdin, A. E. (2005). Informant discrepancies in the assessment of childhood psychopathology: A critical review, theoretical framework, and recommendations for further study. Psychological Bulletin, 131(4), 483-509. doi:10.1037/0033-2909.131.4.483

Gagnon, C., Vitaro, F., \& Tremblay, R. E. (1992). Parent-teacher agreement on kindergarteners' behavior problems: A research note. Journal of Child Psychology and Psychiatry, 33(7), 1255-1261.

Gagnon, S. G., Nagle, R. J., \& Nickerson, A. B. (2007). Parent and teacher ratings of peer interactive play and social-emotional development of preschool children at risk. Journal of Early Intervention, 29(3), 228-242. doi:10.1177/105381510702900303

Gimpel, G. A., \& Holland, M. L. (2003). Emotional and behavioral problems of young children: Effective interventions in the preschool and kindergarten years. New York: Guilford Press.

Goodman, R. (2001). Psychometric properties of the Strengths and Difficulties Questionnaire. Journal of American and Academic Child and Adolescent Psychiatry, 40(11), 1337-1345.

Grietens, H., Onghena, P., Prinzie, P., Gadeyne, E., Van Assche, V., Ghesquière, P., \& Hellinckx, W. (2004). Comparison of mothers', fathers', and teachers' reports on problem behavior in 5- to 6-years-old children. Journal of Psychopathology and Behavioral Assessment, 26(2), 137-146. doi:10.1023/ B:JOBA.0000013661.14995.59

Gross, D., Fogg, L., Garvey, C., \& Julion, W. (2004). Behavior problems in young children: An analysis of cross-informant agreements and disagreements. Research in Nursing \& Health, 27, 413-425. doi:10.1002/nur.20040

Herrera, M., \& Little, E. (2005). Behaviour problems across home and kindergarten in an Australian sample. Australian Journal of Educational \& Developmental Psychology, 5, 77-90.

Hinshaw, S. P., Han, S. S., Erhardt, D., \& Huber, A. (1992). Internalizing and externalizing behavior problems in preschool children: Correspondence among parent and teacher ratings and behavior observations. Journal of Clinical Child Psychology, 21(2), 143-150.

Hundert, J., Morrison, L., Mahoney, W., Mundy, F., \& Vernon, M. L. (1997). Parent and teacher assessments of the developmental status of children with severe, mild/moderate, or no developmental disabilities. Topics in Early Childhood Special Education, 17(4), 419-434.

Kamphaus, R. W., \& Frick, P. J. (1996). Clinical assessment of child and adolescent personality and behavior. Boston: Allyn and Bacon.

Keenan, K., \& Wakschlag, L. S. (2000). More than the terrible twos: The nature and severity of behavior problems in clinic-referred preschool children. Journal of Abnormal Child Psychology, 28(1), 33-46.
Keogh, B. K., \& Bernheimer, L. P. (1998). Concordance between mothers' and teachers' perceptions of behavior problems of children with developmental delays. Journal of Emotional and Behavioral Disorders, 6(1), 33-41.

Klyce, D., Conger, A. J., Conger, J. C., \& Dumas, J. E. (2011). Measuring competence and dysfunction in preschool children: Source agreement and component structure. Journal of Child and Family Studies, 20, 503-510. doi: 10.1007/s10826-0109417-0

Kroes, G. (2006). The perception of child problem behavior. The role of informant personality and context (Doctoral dissertation, Radboud Universiteit Nijmegen, Netherlands). Retrieved from http://dare.ubn.kun.nl/dspace/bitstream/2066/55482/1/55482. pdf

Lane, K. L., Stanton-Chapman, T., Jamison, K. R., \& Phillips, A. (2007). Teacher and parent expectations of preschoolers' behavior: Social skills necessary for success. Topics in Early Childhood Special Education, 27(2), 86-97. doi:10.1177/027 11214070270020401

Liu, J., Cheng, H., \& Leung, P. W. (2011). The application of the Preschool Child Behavior Checklist and the CaregiverTeacher Report Form to Mainland Chinese children: Syndrome structure, gender differences, country effects, and interinformant agreement. Journal of Abnormal Child Psychology, 39, 251-264. doi: 10.1007/s10802-010-9452-8

Major, S. (2011). Avaliação de aptidões sociais e problemas de comportamento em idade pré-escolar: Retrato das crianças portuguesas (Unpublished doctoral dissertation). Retrieved from https://estudogeral.sib.uc.pt/bitstream/10316/17774/5/ Tese_Sofia\%20Major.pdf

Mashburn, A. J., Hamre, B. K., Downer, J. T., \& Pianta, R. C. (2006). Teacher and classroom characteristics associated with teachers' ratings of prekindergartners' relationships and behaviors. Journal of Psychoeducational Assessment, 24(4), 367-380. doi:10.1177/0734282906290594

Merrell, K. W. (2002). Preschool and Kindergarten Behavior Scales. ( $2^{\text {nd }}$ Ed.). Austin, TX: PRO-ED..

Milfort, R., \& Greenfield, D. B. (2002). Teacher and observer ratings of head start children's social skills. Early Childhood Research Quarterly, 17, 581-595. doi:10.1016/s0885-2006(02)00190-4

Miner, J. L., \& Clarke-Stewart, K. A. (2008). Trajectories of externalizing behavior from age 2 to age 9: Relations with gender, temperament, ethnicity, parenting, and rater. Developmental Psychology, 44(3), 771-786. doi:10.1037/00121649.44.3.771

Murray, D. W., Kollins, H. S., Hardy, K. K., Abikoff, H. B., Swanson, J. M., Cunningham, C., . . , \& Chuang, S. Z. (2007). Parent versus teacher ratings of attention-deficit/hyperactivity disorder symptoms in the preschoolers with Attention-Deficit/ Hyperactivity Disorder treatment study (PATS). Journal of Child and Adolescent Psychopharmacology, 17(5), 605-619. doi: 10.1089/cap.2007.0060

Offord, D. R., Boyle, M. H., Racine, Y., Szatmari, P., Fleming, J. E., Sanford, M., \& Lipman, E. L. (1996). Integrating assessment data from multiple informants. Journal of American and Academic Child and Adolescent Psychiatry, 35(8), 1078-1085.

Ordway, M. R. (2011). Depressed mothers as informants on child behavior: Methodological issues. Research in Nursing \& Health, 34(6), 520-532. doi:10.1002/nur.20463 
Phares, V., \& Compas, B. E. (1992). The role of fathers in child and adolescent psychopathology: Make room for daddy. Psychological Bulletin, 111(3), 387-412.

Phares, V., Fields, S., Kamboukos, D., \& Lopez, E. (2005). Still looking for poppa. American Psychologist, 60(7), 735-736. doi:10.1037/0003-066X.60.7.735

Phillips, B. M., \& Lonigan, C. J. (2010). Child and informant influences on behavioral ratings of preschool children. Psychology in the Schools, 47(4), 374-390. doi:10.1002/ pits. 20476

Renk, K. (2005). Cross-informant ratings of the behavior of children and adolescents: The "Gold Standard". Journal of Child and Family Studies, 14(4), 457-468. doi:10.1007/s10826-0057182-2

Renk, K., \& Phares, V. (2004). Cross-informant ratings of social competence in children and adolescents. Clinical Psychology Review, 24, 239-254. doi:10.1016/j.cpr.2004.01.004

Seabra-Santos, M. J., \& Gaspar, M. F. (2012). Pais, educadores e testes: Estão de acordo na avaliação de aptidões de crianças pré-escolares? Psicologia: Reflexão e Crítica, 25(2), 203-211.

Stanger, C., \& Lewis, M. (1993). Agreement among parents, teachers, and children on internalizing and externalizing behavior problems. Journal of Clinical Child Psychology, 22(1), 107-115.

Stefan, C. A., Balaj, A., Porumb, M., Albu, M., \& Miclea, M. (2009). Preschool screening for social and emotional competencies Development and psychometric properties. Cognition, Brain \& Behavior. An Interdisciplinary Journal, 13(2), 121-146.
Stone, L. L., Otten, R., Engels, R. C. M., Vermulst, A. A., \& Janssens, J. M. A. (2010). Psychometric properties of the parent and teacher versions of the Strengths and Difficulties Questionnaire for 4- to 12-year-olds: A review. Clinical Child Family Psychology Review, 13, 254-274. doi: 10.1007/s10567010-0071-2

Tarren-Sweeney, M. J., Hazell, P. L., \& Carr, V. J. (2004). Are foster parents reliable informants of children's behaviour problems? Child: Care, Health \& Development, 30(2), 167175. doi:10.1111/j.1365-2214.2003.00407.x

Treutler, C. M., \& Epkins, C. C. (2003). Are discrepancies among child, mother, and father reports on children's behavior related to parents' psychological symptoms and aspects of parentchild relationships? Journal of Abnormal Child Psychology, 31(1), 13-27.

Verhulst, F. C., \& Akkerhuis, G. W. (1989). Agreement between parents' and teachers' ratings of behavioral/emotional problems of children aged 4-12. Journal of Child Psychology and Psychiatry, 30(1), 123-136.

Vitaro, F., Gagnon, C., \& Tremblay, R. E. (1991). Teachers' and mothers' assessment of children's behaviors from kindergarten to grade two: Stability and change within and across informants. Journal of Psychopathology and Behavioral Assessment, 13(4), 325-343.

Winsler, A., \& Wallace, G. L. (2002). Behavior problems and social skills in preschool children: Parent-teacher agreement and relations with classroom observations. Early Education \& Development, 13(1), 41-58. doi:10.1207/s15566935eed1301_3 